\title{
Programmed cell death 1 expression is associated with inferior survival in patients with primary central nervous system lymphoma
}

\author{
Hyunsoo Cho ${ }^{1,2, *}$, Se Hoon Kim ${ }^{3, *}$, Soo-Jeong Kim ${ }^{1}$, Jong Hee Chang ${ }^{4}$, Woo-Ick Yang ${ }^{3}$, \\ Chang-Ok Suh ${ }^{5}$, Yu Ri Kim ${ }^{1}$, Ji Eun Jang ${ }^{1}$, June-Won Cheong ${ }^{1}$, Yoo Hong Min ${ }^{1}$ and \\ Jin Seok Kim ${ }^{1}$ \\ ${ }^{1}$ Division of Hematology, Department of Internal Medicine, Yonsei University College of Medicine, Severance Hospital, Seoul \\ 03722, Republic of Korea \\ ${ }^{2}$ Graduate School of Medical Science and Engineering, Korea Advanced Institute of Science and Technology, Daejeon 34141, \\ Republic of Korea \\ ${ }^{3}$ Department of Pathology, Yonsei University College of Medicine, Severance Hospital, Seoul 03722, Republic of Korea \\ ${ }^{4}$ Department of Neurosurgery, Yonsei University College of Medicine, Severance Hospital, Seoul 03722, Republic of Korea \\ ${ }^{5}$ Department of Radiation Oncology, Yonsei University College of Medicine, Severance Hospital, Seoul 03722, Republic of \\ Korea \\ * These authors have contributed equally to this work
}

Correspondence to: Jin Seok Kim, email: hemakim@yuhs.ac

Keywords: primary central nervous system lymphoma, programmed cell death 1, programmed cell death-ligand 1, programmed cell death-ligand 2, prognosis

Received: June 12,2017 Accepted: July 13,2017 Published: August 14, 2017

Copyright: Cho et al. This is an open-access article distributed under the terms of the Creative Commons Attribution License 3.0 (CC BY 3.0), which permits unrestricted use, distribution, and reproduction in any medium, provided the original author and source are credited.

\section{ABSTRACT}

Programmed cell death 1 (PD-1) and its ligands PD-L1/PD-L2 have been shown to mediate immune evasion in various cancers, but their prognostic implications in patients with primary central nervous system lymphoma (PCNSL) are poorly understood. Therefore, we analyzed 76 PCNSL patients at initial diagnosis who were treated homogenously with high-dose methotrexate-based chemotherapy, and evaluated the prognostic roles of high immunohistochemical PD-1, PD-L1, and PD-L2 expression. The cut-off values for high PD-1 ( $\geq 70$ cells/high power field [HPF]), PD-L1 ( $\geq 100$ cells/HPF), and PD-L2 ( $\geq 100$ cells/HPF) were determined by the area under the receiver operating characteristic curve. Expression of PD-1, PD-L1, and PD-L2 was high in $7.9 \%, 13.2 \%$, and $42.1 \%$ patients, respectively. High PD-1, $(P=0.007)$ and Memorial Sloan Kettering Cancer Center (MSKCC) prognostic scoring $(P=0.019)$ were independently associated with inferior overall survival on multivariate analysis. High PD-1 also remained an independent prognostic factor for inferior progression-free survival $(P=\mathbf{0 . 0 2 8})$, as did MSKCC prognostic scoring $(P=\mathbf{0 . 0 4 1})$ on multivariate analysis. However, there were no differences in survival according to the expression levels of PD-L1/PD-L2 in PCNSL tumor microenvironment. Our results suggest that PD-1 may be considered a biomarker and potential therapeutic target in PCNSL.

\section{INTRODUCTION}

Primary central nervous system (CNS) lymphoma (PCNSL) is an extranodal non-Hodgkin lymphoma (NHL) confined to the CNS, mostly diffuse large B-cell lymphoma (DLBCL) [1]. Currently, the International Extranodal Lymphoma Study Group (IELSG) and
Memorial Sloan Kettering Cancer Center (MSKCC) prognostic scoring systems are the best available clinical tools to risk-stratify patients with PCNSL [2, $3]$. However, these prognostic scores do not take into account underlying tumor biological factors, such as tumor microenvironment of PCNSL. Several biomarkers for PCNSL have been suggested, including multiple 
myeloma oncogene 1 /interferon regulatory factor 4 (MUM1/IRF4), B-cell lymphoma-6 (BCL-6) and CD68, but their major limitation is that it is difficult to use those biomarkers as a potential therapeutic target [4-6]. Therefore, discovering a novel biomarker that supplements IELSG and MSKCC prognostic scoring system and that can be used as a therapeutic target is desperately needed to improve prognostication and survival of patients with PCNSL.

Tumor microenvironment per se is important for PCNSL development and progression as well as other malignancies [7]. This is also supported by the study that showed characteristic infiltration by tumor infiltrating lymphocytes in perivascular microenvironment of PCNSL, which was associated with survival [8]. Albeit the immunological role of T-cell infiltration in PCNSL is unclear, emerging data are suggesting that tumors have developed evasion mechanisms that exploit immune checkpoints to overcome antitumor immunity [9].

The immune checkpoint molecule programmed cell death 1 (PD-1) and its ligands PD-L1 and PD-L2 have been shown to play key roles in inhibiting $\mathrm{T}$ cell activity in the tumor microenvironment, not only in solid cancers but also in hematologic malignancies [10]. PD-1 and its ligands have been highlighted because their blockade showed outstanding clinical responses in advanced hematologic malignancies [11].

Importantly, PCNSL exhibited frequent 9p24.1/ PD-L1/PD-L2 copy number alterations [9] and even PD-1 blockade demonstrated clinical activity in relapsed/ refractory PCNSL [12], although only small number of patients were evaluated. Based on these findings, we sought to evaluate PD-1, PD-L1, and PD-L2 expressions in immunocompetent PCNSL patients at diagnosis, as well as their prognostic implication.

\section{RESULTS}

\section{Patients' characteristics}

Baseline patient characteristics according to PD-1, PD-L1, and PD-L2 expression levels are summarized in Table 1. The median follow-up duration for all patients was 20.2 (range, 2.2-128.5) months, and the median follow-up duration for surviving patients was 31.9 (range, 2.4-128.5) months. Serum Epstein-Barr virus (EBV) DNA was detected in $16(21.1 \%)$ patients (median 9,200 [range 1,944-88,000] copies/mL blood).

All the patients received high-dose-methotrexate (HD-MTX)-based chemotherapy as an initial treatment and there were no significant differences in survival according to the initial HD-MTX-based chemotherapy regimens patients received. Sixteen (21.1\%) patients received consolidative upfront autologous stem-cell transplantation (ASCT) after a median of 4 cycles (range 2-4) of HD-MTX-based chemotherapy. Ten (16.7\%) patients received salvage ASCT. Busulfan plus thiotepa conditioning regimen was used for upfront or salvage ASCT. Twelve (15.8\%) patients received consolidation whole-brain radiotherapy (WBRT, median 40.5 [range 25.2-45.0] Gy), and 14 (18.4\%) patients underwent salvage WBRT (median 41.4 [range 36.0-54.0] Gy).

Patients did not reach median Overall survival (OS), and the median progression-free survival (PFS) was 18.4 months (95\% CI, 9.9-26.8). The two-year OS and PFS rates were $76.3 \%$ and $45.4 \%$, respectively.

\section{Immunohistochemical PD-1, PD-L1 and PD-L2 expression}

Overall, the median PD-1 positive cells/high power field (HPF) was 0 (range, 0-240). Among 76 patients, 41 $(53.9 \%)$ patients did not show PD-1 positive cells/HPF. However, remaining 35 (46.1\%) patients expressed at least one PD-1 positive cells/HPF. The median positive cells/HPF was 10 (range, 0-300) for PD-L1 and 70 (range, 0-300) for PD-L2. Twenty-two (28.9\%) and 12 (15.8\%) patients did not express PD-L1 and PD-L2, respectively. Remaining 54 (71.1\%) and 64 (84.2\%) patients expressed at least one PD-L1 and PD-L2 positive cells/HPF, respectively. We stratified patients into high or low $\mathrm{PD}$ 1, PD-L1, and PD-L2 expression groups according to the area under curve (AUC)-based cut-off values. Six (7.9\%) patients were stratified into the high PD-1 expression group. Ten $(13.2 \%)$ and $32(42.1 \%)$ patients were stratified into the high PD-L1 and high PD-L2 expression groups, respectively. Representative immunohistochemical stains of PD-1 (Figure 1b), PD-L1 (Figure 1c), and PD-L2 (Figure 1d) are shown in Figure 1 with unstained control (Figure 1a).

\section{Prognostic factors for survival}

On univariate analysis, age $>60$ years and Eastern Cooperative Oncology Group (ECOG) performance status (PS) $\geq 2$, MSKCC prognostic scoring, IELSG prognostic scoring, and high PD-1 expression level were associated with inferior OS. MSKCC prognostic scoring, patients who did not achieve complete response at initial interim response (non-CR1), patient who did not receive upfront ASCT, and high PD-1 level were associated with inferior PFS also in univariate analysis. On multivariate analysis, high PD-1 (hazard ratio (HR): 4.95, 95\% confidence interval (CI): 1.54-15.86, $P=0.007)$ and MSKCC prognostic scoring (HR: 2.56, 95\% CI: 1.17-5.64, $P=$ 0.019 ) were independently associated with inferior OS. High PD-1 also remained an independent prognostic factor for inferior PFS, (HR 2.73, 95\% CI: 1.12-6.69, $P=0.028$ ) as did MSKCC prognostic scoring (HR: 1.56, 95\% CI: $1.09-2.45, P=0.041)$ on multivariate analysis. However, PD-L1 and PD-L2 expression levels were not prognostic in our cohort (Table 2). 
Table 1: Baseline characteristics of all patients and subgroups according to the levels of PD-1, PD-L1, and PD-L2 ex pression

\begin{tabular}{|c|c|c|c|c|c|c|c|c|c|c|}
\hline \multirow{3}{*}{$\begin{array}{l}\text { Clinical features, } \\
n(\%)\end{array}$} & \multirow{3}{*}{$\begin{array}{c}\text { Entire cohort } \\
(n=76)\end{array}$} & \multicolumn{9}{|c|}{ Subgroups } \\
\hline & & \multicolumn{2}{|c|}{ PD-1 } & \multirow{2}{*}{$P$} & \multicolumn{2}{|c|}{ PD-L1 } & \multirow{2}{*}{$P$} & \multicolumn{2}{|c|}{ PD-L2 } & \multirow{2}{*}{$P$} \\
\hline & & Low $(n=70)$ & $\operatorname{High}(n=6)$ & & Low $(n=66)$ & High $(n=10)$ & & Low $(n=44)$ & $\operatorname{High}(n=32)$ & \\
\hline Median age, year & $57(33-79)$ & $56(33-79)$ & $59(34-68)$ & & $56(33-79)$ & $59(34-64)$ & & $58(36-79)$ & $56(33-74)$ & \\
\hline Age $>60$ & $30(39.5)$ & $28(40.0)$ & $2(33.3)$ & 0.556 & $28(42.4)$ & $2(20.0)$ & 0.158 & $27(61.4)$ & $19(59.4)$ & 0.524 \\
\hline Male gender & $39(51.3)$ & $36(51.4)$ & $3(50.0)$ & 0.637 & $35(53.0)$ & $4(40.0)$ & 0.334 & $23(52.3)$ & $16(50.0)$ & 0.514 \\
\hline ECOG PS $\geq 2$ & $35(46.1)$ & $34(48.6)$ & $1(16.7)$ & 0.209 & $29(43.9)$ & $6(60.0)$ & 0.271 & $17(38.6)$ & $18(56.3)$ & 0.099 \\
\hline $\begin{array}{l}\text { Elevated serum } \\
\text { LDH }\end{array}$ & $34(44.7)$ & $33(47.1)$ & $1(16.7)$ & 0.216 & $29(43.9)$ & $5(50.0)$ & 0.489 & $20(45.5)$ & $14(43.8)$ & 0.535 \\
\hline Deep lesion & $51(67.1)$ & $47(67.1)$ & $4(66.7)$ & 0.649 & $43(65.2)$ & $8(80.0)$ & 0.293 & $27(61.4)$ & $24(75.0)$ & 0.158 \\
\hline $\begin{array}{l}\text { Elevated CSF } \\
\text { protein }\end{array}$ & $30 / 66(39.4)$ & 28/61 (40.0) & $2 / 5(33.3)$ & 0.587 & 27/57 (40.9) & $3 / 9(30.0)$ & 0.339 & 18/39 (40.9) & $12 / 27(37.5)$ & 0.546 \\
\hline $\begin{array}{l}\text { Positive serum } \\
\text { EBV }\end{array}$ & $16(21.1)$ & $14(20.0)$ & $2(33.3)$ & 0.371 & $14(21.2)$ & $2(20.0)$ & 0.648 & $11(25.0)$ & $5(15.6)$ & 0.242 \\
\hline $\begin{array}{l}\text { Histology, } \\
\text { DLBCL/PTCL }\end{array}$ & $75(98.7) / 1(1.3)$ & $69(98.6) / 1(1.4)$ & $6(100.0) / 0(0.0)$ & 0.921 & $65(98.5) / 1(1.5)$ & $10(100.0) / 0(0.0)$ & 0.868 & $43(97.7) / 1(2.3)$ & $32(100.0) / 0(0.0)$ & 0.579 \\
\hline IELSG & & & & 0.257 & & & 0.507 & & & 0.097 \\
\hline Low (0-1) & $12(15.8)$ & $10(14.3)$ & $2(33.3)$ & & $10(15.2)$ & $2(20.0)$ & & $8(18.2)$ & $4(12.5)$ & \\
\hline $\begin{array}{l}\text { Intermediate } \\
(2-3)\end{array}$ & $46(60.5)$ & $43(61.4)$ & $3(50.0)$ & & $41(62.1)$ & $5(50.0)$ & & $29(65.9)$ & $17(53.1)$ & \\
\hline High (4-5) & $8(10.5)$ & $8(11.4)$ & $0(0.0)$ & & $6(9.1)$ & $2(20.0)$ & & $2(4.5)$ & $6(18.8)$ & \\
\hline Missing & $10(13.2)$ & $9(12.9)$ & $1(16.7)$ & & $9(13.6)$ & $1(10.0)$ & & $5(11.4)$ & $5(15.6)$ & \\
\hline MSKCC & & & & 0.180 & & & 0.433 & & & 0.208 \\
\hline Low (0) & $15(19.7)$ & $13(18.6)$ & $2(33.3)$ & & $13(19.7)$ & $2(20.0)$ & & $9(20.5)$ & $6(18.8)$ & \\
\hline Intermediate (1) & $32(42.1)$ & $29(41.4)$ & $3(50.0)$ & & $27(40.9)$ & $5(50.0)$ & & $21(47.7)$ & $11(34.4)$ & \\
\hline High (2) & $29(38.2)$ & $28(40.0)$ & $1(16.7)$ & & $26(39.4)$ & $3(30.0)$ & & $14(31.8)$ & $15(46.9)$ & \\
\hline $\begin{array}{l}\text { Initial treatment } \\
\text { MVD/MVP }\end{array}$ & $41(53.9) / 35(46.1)$ & $37(52.9) / 33(47.1)$ & $4(66.7) / 2(33.3)$ & 0.416 & $40(60.6) / 26(39.4)$ & $1(10.0) / 9(90.0)$ & 0.003 & $25(56.8) / 19(43.2)$ & $16(50.0) / 16(50.0)$ & 0.361 \\
\hline Non-CR1 & $37(48.7)$ & $34(48.6)$ & $3(50.0)$ & 0.389 & $31(47.0)$ & $6(60.0)$ & 0.562 & $22(50.0)$ & $15(46.9)$ & 0.380 \\
\hline Upfront ASCT & $16(21.1)$ & $15(21.4)$ & $1(16.7)$ & 0.629 & $13(19.7)$ & $3(30.0)$ & 0.352 & $9(20.5)$ & 7 (21.9) & 0.550 \\
\hline Salvage ASCT & $10(13.2)$ & $8(11.4)$ & $2(33.3)$ & 0.176 & $9(13.6)$ & $1(10.0)$ & 0.609 & $7(15.9)$ & $3(9.4)$ & 0.318 \\
\hline $\begin{array}{l}\text { Consolidation } \\
\text { WBRT }\end{array}$ & $12(15.8)$ & $8(11.4)$ & $4(66.7)$ & 0.657 & $5(7.6)$ & $7(70.0)$ & 0.495 & $7(15.9)$ & $5(15.6)$ & 0.384 \\
\hline Salvage WBRT & $14(18.4)$ & $13(18.6)$ & $1(16.7)$ & 0.696 & $12(18.2)$ & $2(20.0)$ & 0.590 & $9(20.5)$ & $5(15.6)$ & 0.411 \\
\hline
\end{tabular}

Abbreviations: ECOG, Eastern Cooperative Oncology Group; PS, performance status; LDH, lactate dehydrogenase; CSF, cerebrospinal fluid; EBV, Epstein-Barr virus; DLBCL, diffuse large B-cell lymphoma; PTCL, peripheral T-cell lymphoma; IELSG, International Extranodal Lymphoma Study Group; MSKCC, Memorial Sloan Kettering Cancer Center; MVD, methotrexate, vincristine and dexamethasone; MVP, methotrexate, vincristine, procarbazine and dexamethasone; CR1, complete response after first two cycles of chemotherapy; ASCT, autologous stemcell transplantation; WBRT, whole-brain radiotherapy.

\section{Survival according to the expression levels of PD-1, PD-L1, and PD-L2}

Patients with high expression of PD-1 showed significantly shorter 2-year OS and PFS of $33.3 \%$ and $0.0 \%$, in comparison to $81.2 \%$ and $50.5 \%$ for those with low expression of PD-1 ( $P=0.008$ for OS; Figure 2a, and $P=0.037$ for PFS; Figure $2 b$ ), respectively.

Regarding PD-1 ligands, there were no differences in survival according to the levels of PD-L1 and PDL2 expression (Figure 2c-2d for PD-L1 and Figure 2e$2 \mathrm{f}$ for PD-L2, respectively). Two-year survival rates of patients according to the PD-1, PD-L1, and PD-L2 expression status are provided in Table 3 and associations of PD-1, PD-L1, and PD-L2 expression levels with survival are summarized in Table 4.

\section{Subgroup analysis of patients without upfront ASCT according to PD-1 expression level}

As high PD-1 expression was independently associated with inferior survival in our whole patients, we performed subgroup analysis in patients who did not undergo upfront ASCT according to the level of PD-1 
expression to consider further confounding variables. In $60(78.9 \%)$ patients who did not undergo upfront ASCT, high PD-1 expression was observed in $5(8.3 \%)$ patients and they tended to associate with inferior survival (Figure $3 \mathrm{a}$ for OS and Figure $3 \mathrm{~b}$ for PFS) although not statistically significant.

\section{Survival of patients with high PD-1 expression}

We further analyzed survival of patients with high PD-1 expression to better characterize the patients with high PD-1 expression in PCNSL tumor microenvironment. The median survival of patients with high PD-1 expression was only 15.8 months (95\% CI: 11.7-19.9) for OS and 10.4 months (95\% CI: 3.3-17.5) for PFS. However, the median OS of patients with low PD-1 expression was not reached, and median PFS was 24.7 months (95\%
CI: 9.8-39.5) in patients with low PD-1. All the PCNSL patients with high PD-1 expression had DLBCL histology and two out of six patients showed positive serum EBV DNA among high PD-1 patients. Among six patients with high PD-1 expression, three patients achieved CR1 and five patients achieved CR after completion of primary chemotherapy.

Among these high PD-1 expressing patients, four patients died of disease progression, although four of whom received WBRT consolidation (median 4.0 [range 30.6-54.0] Gy) and one patient received upfront ASCT consolidation. Among four patients with high PD-1 who died of progression, two patients were intermediate-risk, one patient was low-risk, and one patient had missing data according to the IELSG scoring. Regarding MSKCC scoring, two patients belonged to low-risk, and the remaining two patients belonged to intermediate and high-
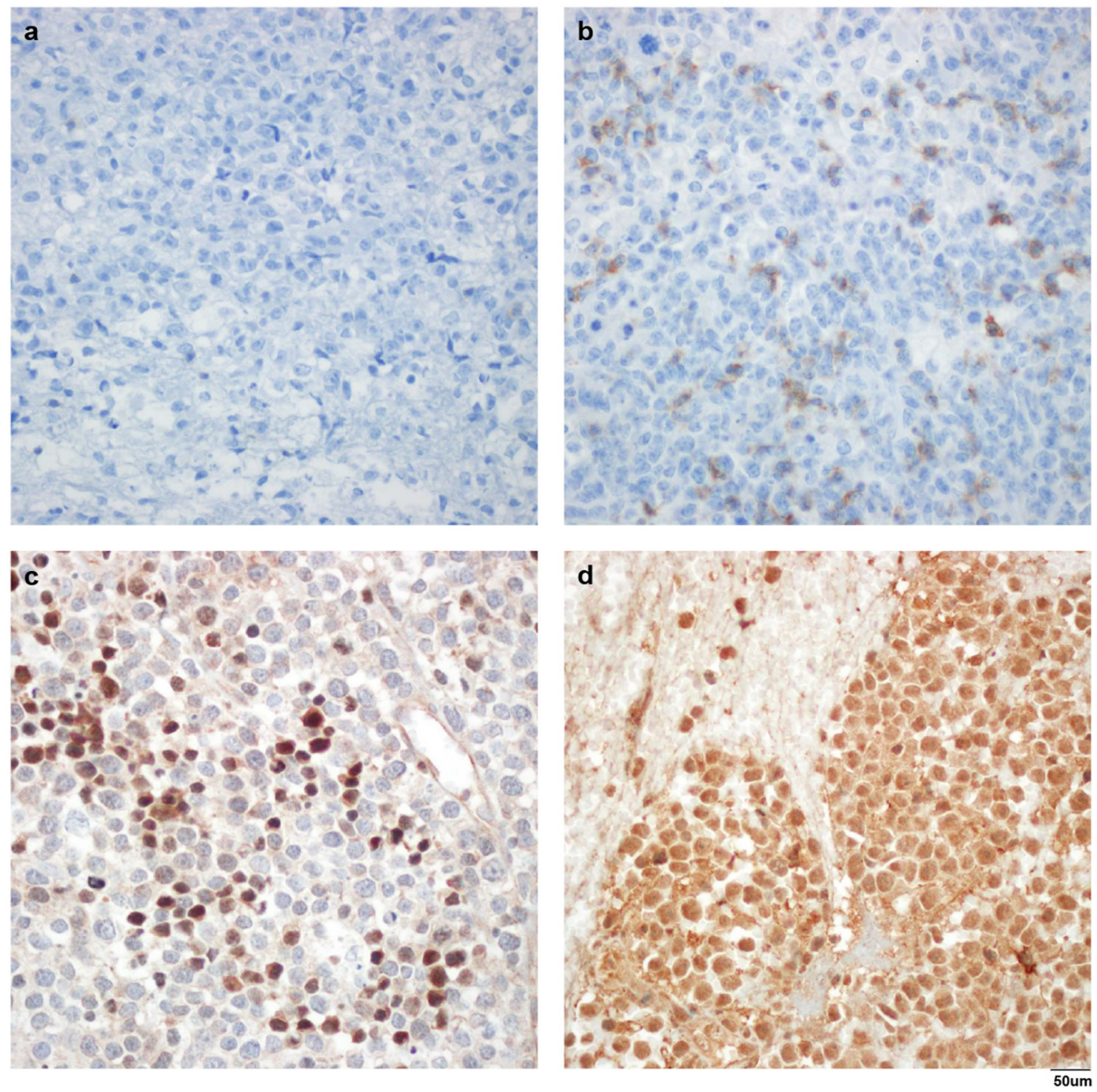

Figure 1: Representative immunohistochemical stains in formalin-fixed and paraffin-embedded samples. Negative control (a), high PD-1 expression (b), PD-L1 (c), and PD-L2 (d) with anti-PD-1 antibody (NAT105, a and b), anti-PD-L1 antibody $(\mathrm{ab58810}, \mathrm{c})$, and anti-PD-L2 antibody (MIH18, d). Magnification, $\times 400$. 
Table 2: Univariate and multivariate analyses for overall survival (OS) and progression-free survival (PFS)

\begin{tabular}{|c|c|c|c|c|c|c|}
\hline \multirow{2}{*}{ Clinical features } & \multicolumn{2}{|c|}{ Univariate analysis } & \multicolumn{4}{|c|}{ Multivariate analysis } \\
\hline & $P$ for $O S$ & $P$ for PFS & HR $(95 \%$ CI $)$ for OS & $P$ & HR (95\% CI) for PFS & $P$ \\
\hline Age $>60$ years & 0.038 & 0.159 & & & & \\
\hline Male gender & 0.140 & 0.707 & & & & \\
\hline ECOG PS $\geq 2$ & 0.047 & 0.353 & & & & \\
\hline Elevated LDH & 0.209 & 0.103 & & & & \\
\hline Deep brain lesion & 0.696 & 0.361 & & & & \\
\hline Elevated CSF protein & 0.074 & 0.064 & & & & \\
\hline Positive serum EBV & 0.148 & 0.173 & & & & \\
\hline Non-CR1 & 0.791 & 0.005 & & & & \\
\hline Non-upfront ASCT & 0.238 & 0.040 & & & & \\
\hline MSKCC scoring & 0.029 & 0.046 & $2.56(1.17-5.64)$ & 0.019 & $1.56(1.09-2.45)$ & 0.041 \\
\hline IELSG scoring & 0.036 & 0.487 & & & & \\
\hline PD- $1 \geq 70$ cells/HPF & 0.018 & 0.043 & $4.95(1.54-15.86)$ & 0.007 & $2.73(1.12-6.69)$ & 0.028 \\
\hline PD-L1 $\geq 100$ cells/HPF & 0.764 & 0.793 & & & & \\
\hline PD-L2 $\geq 100$ cells/HPF & 0.306 & 0.940 & & & & \\
\hline
\end{tabular}

OS, overall survival; PFS, progression-free survival; HR, hazard ratio; CI, confidence interval; ECOG, Eastern Cooperative Oncology Group; PS, performance status; LDH, lactate dehydrogenase; CSF, cerebrospinal fluid; EBV, Epstein-Barr virus; CR1, complete response after first two cycles of chemotherapy; ASCT, autologous stem-cell transplantation; HPF, high power field; MSKCC, Memorial Sloan Kettering Cancer Center; IELSG, International Extranodal Lymphoma Study Group.

risk, respectively. Additional characteristics of patients with high PD-1 expression are provided in Table 5.

\section{Correlation of PD-1 expression with PD-L1 and PD-L2 expression levels}

The association of PD-1 expression with PD-L1 and PD-L2 expression in tumor microenvironment of PCNSL is shown in Table 6. The level of PD-1 expression was significantly associated with the level of PD-L1 $(P=$ $0.020)$ and PD-L2 $(P<0.001)$, respectively (Table 6$)$.

\section{DISCUSSION}

In this study, we found that high immunohistochemical PD-1 expression in biopsy specimen of patients with PCNSL at diagnosis was significantly associated with inferior OS and PFS.

Much information has been accumulated regarding the pathogenesis and tumor microenvironment of PCNSL [13]. However, in addition to the rarity of the disease, obtaining adequate numbers of specimens is difficult and thus prognostic roles of studied biomarkers have been unclear. Therefore, IELSG or MSKCC scoring is thus far considered the best available clinical tool for risk-stratifying PCNSL patients [2, 3], although biological prognostic markers that also consider the tumor microenvironment and that can be used as a therapeutic target are needed to improve prognostication and survival of patients with PCNSL. Although our group recently reported prognostic importance of $\mathrm{CD} 68$ expression in PCNSL microenvironment [6], the major limitation was regarding therapeutic implication as it is difficult to modulate immune response via $\mathrm{CD} 68$ positive macrophages in PCNSL tumor microenvironment.

In this regard, PD-1 is an attractive emerging therapeutic target because it has been shown to be expressed in various cancers as well as in hematologic malignancies [14, 15]. Regarding PD-1 expression in PCNSL, Berghoff et al. [16] were among the first to demonstrate PD-1 and PD-L1 expression in PCNSL. Of 20 PCNSL patient specimens, $2(10.0 \%)$ showed moderate intensity and $1(5.0 \%)$ had high intensity. Besides, Four et al. [17] reported expression of high PD-1 (2+) in 6.2\% of patients with PCNSL. The proportions of high PD-1 expressing patients are similar to our study, as $7.9 \%$ of our patients expressed high PD-1. Compared to those studies, our study recruited more number of patients who were initially homogenously treated with HD-MTX-based chemotherapy. We also performed subgroup analysis 
according to the upfront ASCT, as the role of upfront ASCT in high-risk patients with PCNSL was emphasized [18].

One of our patient with high PD-1 expression died of progression even after receiving upfront ASCT. Interestingly, the poor prognosis of patients with high PD-1 expression was initially indistinguishable using the IELSG or MSKCC scoring systems; because among four high PD-1 expressing patients who died of disease progression, none of them belonged to the high-risk group according to IELSG scoring and only one of them belonged to the high-risk group regarding MSKCC scoring. However, if we knew that high PD-1 expression predicted poor survival in PCNSL, we would have been able to get more help regarding selection of treatment for these high-risk patients.

There were high expressions of PD-L1 and PD-L2 in $13.2 \%$ and $42.1 \%$ of our cohort, respectively. Regarding PD-L1 expression, our data also showed quite similar rate to that of Four et al. [17], as they reported $18.7 \%$ patients were PD-L1 high. However, these rates are relatively low compared to a recent study of PD-L1 expression in
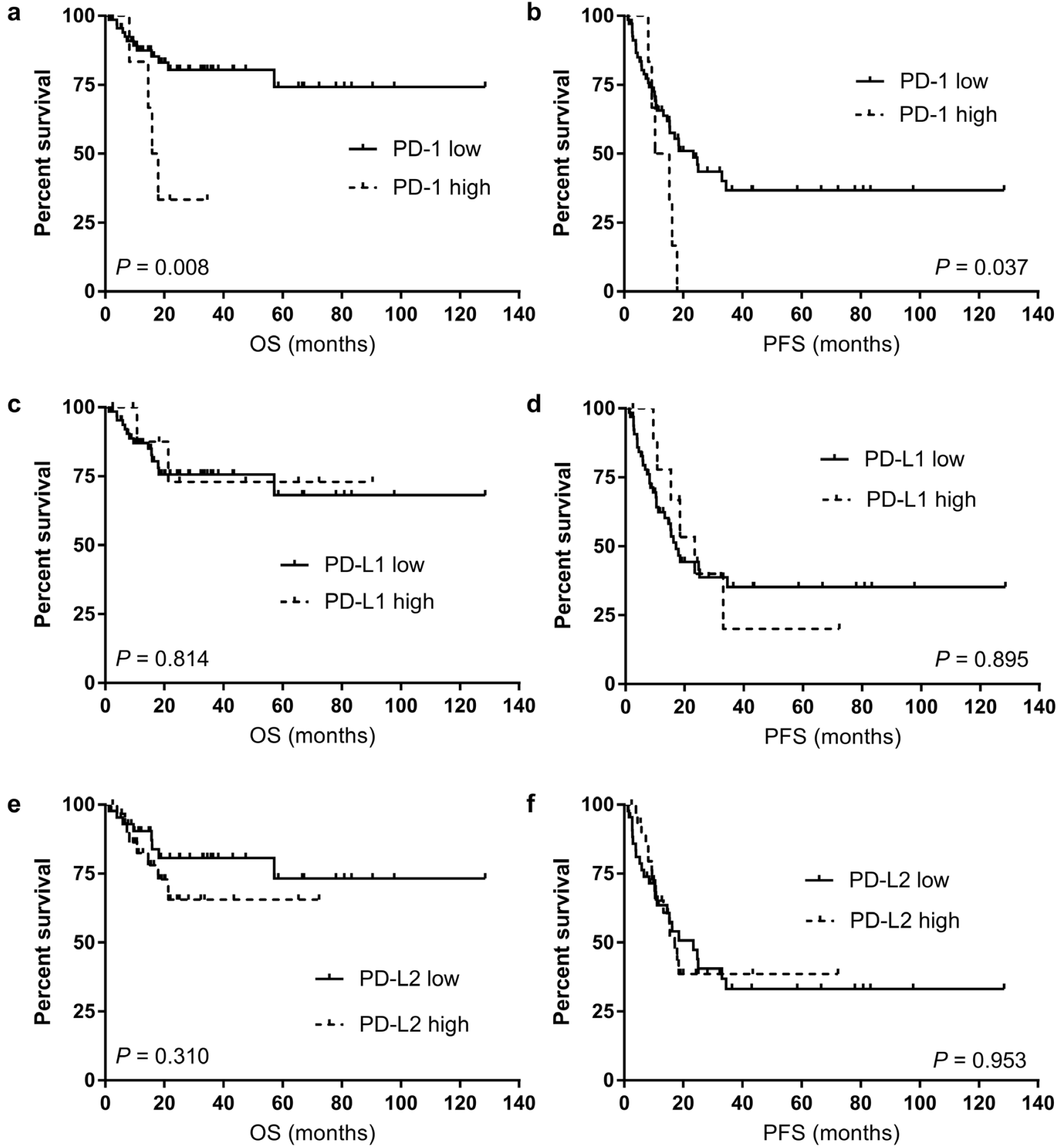

Figure 2: The overall survival (OS) and progression-free survival (PFS) according to the level of PD-1 expression (a for OS and $\mathbf{b}$ for PFS), PD-L1 expression ( $\mathbf{c}$ for OS and $\mathbf{d}$ for PFS), and PD-L2 expression (e for OS and $\mathbf{f}$ for PFS). 
Table 3: Two-year overall survival of patients according to their PD-1, PD-L1, and PD-L2 expression status

\begin{tabular}{|c|c|c|c|c|c|c|c|c|c|c|}
\hline \multirow{3}{*}{ Clinical features, $n$} & \multirow{3}{*}{$\begin{array}{c}\text { Entire cohort } \\
\qquad(n=76)\end{array}$} & \multicolumn{9}{|c|}{ Two-year overall survival (\%) } \\
\hline & & \multicolumn{2}{|c|}{ PD-1 } & \multirow{2}{*}{$P$} & \multicolumn{2}{|c|}{ PD-L1 } & \multirow{2}{*}{$P$} & \multicolumn{2}{|c|}{ PD-L2 } & \multirow{2}{*}{$P$} \\
\hline & & Low $(n=70)$ & High $(n=6)$ & & Low $(n=66)$ & High $(n=10)$ & & Low $(n=44)$ & High $(n=32)$ & \\
\hline \multicolumn{11}{|l|}{ Age } \\
\hline$\leq 60$ & 46 & 88.7 & 50.0 & 0.028 & 84.5 & 85.7 & 0.764 & 88.6 & 76.0 & 0.405 \\
\hline$>60$ & 30 & 66.2 & 0.0 & 0.134 & 61.9 & 0.0 & 0.737 & 66.3 & 28.6 & 0.409 \\
\hline \multicolumn{11}{|l|}{ Gender } \\
\hline Male & 37 & 88.0 & 33.3 & 0.008 & 78.2 & 100.0 & 0.274 & 83.3 & 82.4 & 0.718 \\
\hline Female & 39 & 74.0 & 33.3 & 0.231 & 73.9 & 33.3 & 0.344 & 81.5 & 36.0 & 0.111 \\
\hline \multicolumn{11}{|l|}{ ECOG PS } \\
\hline$<2$ & 41 & 91.6 & 40.0 & 0.004 & 82.0 & 100.0 & 0.377 & 92.4 & 66.1 & 0.070 \\
\hline$\geq 2$ & 35 & 65.9 & 0.0 & 0.199 & 66.2 & 50.0 & 0.981 & 57.9 & 65.9 & 0.572 \\
\hline \multicolumn{11}{|l|}{ Serum LDH } \\
\hline Not elevated & 42 & 71.6 & 40.0 & 0.162 & 66.9 & 66.7 & 0.754 & 75.2 & 50.6 & 0.243 \\
\hline Elevated & 34 & 89.9 & 0.0 & 0.024 & 87.5 & 80.0 & 0.949 & 87.7 & 83.6 & 0.813 \\
\hline \multicolumn{11}{|l|}{ Serum EBV DNA load } \\
\hline Not detected & 60 & 81.7 & 50.0 & 0.143 & 80.4 & 71.4 & 0.086 & 85.4 & 67.4 & 0.248 \\
\hline Detected & 16 & 77.4 & 0.0 & 0.108 & 58.0 & 100.0 & 0.354 & 64.6 & 60.0 & 0.756 \\
\hline \multicolumn{11}{|l|}{ Tumor location } \\
\hline Non-deep lesion & 25 & 77.6 & 50.0 & 0.469 & 70.6 & 100.0 & 0.338 & 77.4 & 71.4 & 0.530 \\
\hline Deep lesion & 51 & 81.4 & 0.0 & 0.009 & 77.9 & 62.5 & 0.730 & 83.1 & 65.5 & 0.368 \\
\hline \multicolumn{11}{|l|}{ CSF protein } \\
\hline Not elevated & 36 & 91.8 & 33.3 & 0.001 & 83.5 & 100.0 & 0.320 & 83.3 & 90.9 & 0.569 \\
\hline Elevated & 30 & 64.3 & 50.0 & 0.673 & 72.3 & 33.3 & 0.440 & 76.6 & 30.3 & 0.249 \\
\hline Not available & 10 & 85.7 & 0.0 & 0.155 & 68.6 & 100.0 & 0.558 & 0.0 & 60.0 & 0.295 \\
\hline \multicolumn{11}{|l|}{ IELSG } \\
\hline Low $(0-1)$ & 12 & 77.1 & 50.0 & 0.426 & 65.6 & 100.0 & 0.377 & 75.0 & 66.7 & 0.743 \\
\hline Intermediate (2-3) & 46 & 88.9 & 33.3 & 0.015 & 82.4 & 100.0 & 0.315 & 83.2 & 85.7 & 0.898 \\
\hline High (4-5) & 8 & 28.1 & N/A & N/A & 66.7 & 0.0 & 0.623 & 50.0 & 31.3 & 0.275 \\
\hline Missing & 10 & 85.7 & 0.0 & 0.155 & 100.0 & 68.6 & 0.558 & 100.0 & 60.0 & 0.295 \\
\hline \multicolumn{11}{|l|}{ MSKCC } \\
\hline Low (1) & 15 & 100.0 & 0.0 & $<0.001$ & 84.6 & 100.0 & 0.571 & 100.0 & 66.7 & 0.070 \\
\hline Intermediate (2) & 32 & 83.9 & 66.7 & 0.531 & 77.6 & 100.0 & 0.321 & 81.5 & 80.0 & 0.858 \\
\hline High (3) & 29 & 62.1 & 0.0 & 0.333 & 67.2 & 0.0 & 0.235 & 65.2 & 33.7 & 0.468 \\
\hline \multicolumn{11}{|l|}{ Initial interim response } \\
\hline CR1 & 39 & 83.6 & 25.0 & 0.007 & 77.6 & 66.7 & 0.839 & 80.8 & 65.6 & 0.605 \\
\hline Non-CR1 & 37 & 77.0 & 50.0 & 0.474 & 73.8 & 75.0 & 0.896 & 80.9 & 64.5 & 0.282 \\
\hline \multicolumn{11}{|l|}{ Upfront ASCT } \\
\hline Yes & 16 & 100.0 & 0.0 & $<0.001$ & 92.3 & 100.0 & 0.395 & 100.0 & 85.7 & 0.738 \\
\hline No & 60 & 74.4 & 40.0 & 0.160 & 53.3 & 71.8 & 0.780 & 76.2 & 54.5 & 0.307 \\
\hline \multicolumn{11}{|l|}{ Consolidation WBRT } \\
\hline Yes & 12 & 66.7 & 50.0 & 0.707 & 70.4 & 0.0 & 0.089 & 79.1 & 0.0 & 0.021 \\
\hline No & 64 & 85.4 & 0.0 & $<0.001$ & 78.0 & 100.0 & 0.159 & 81.9 & 80.9 & 0.962 \\
\hline
\end{tabular}

ECOG, Eastern Cooperative Oncology Group; PS, performance status; LDH, lactate dehydrogenase; EBV, Epstein-Barr virus; CSF, cerebrospinal fluid; IELSG, International Extranodal Lymphoma Study Group; MSKCC, Memorial Sloan Kettering Cancer Center; CR1, complete response after first two cycles of chemotherapy; ASCT, autologous stem-cell transplantation; WBRT, whole-brain radiotherapy; N/A, not available. 
Table 4: Association of PD-1, PD-L1, and PD-L2 expression with survival

\begin{tabular}{|c|c|c|c|c|c|c|c|}
\hline Clinical features & $\begin{array}{c}\text { Total patients } \\
(n)\end{array}$ & $\begin{array}{c}\text { Deaths } \\
(n)\end{array}$ & $\begin{array}{c}\text { Median OS } \\
(95 \% \mathrm{CI})\end{array}$ & $P$ & $\begin{array}{c}\text { Progression } \\
\text { (n) }\end{array}$ & $\begin{array}{c}\text { Median PFS } \\
(95 \% \text { CI })\end{array}$ & $P$ \\
\hline PD-1, cells/HPF & & & & 0.008 & & & 0.037 \\
\hline$<70$ & 70 & 12 & Not reached & & 34 & $24.7(9.8-39.5)$ & \\
\hline$\geq 70$ & 6 & 4 & $15.8(11.7-19.9)$ & & 6 & $10.4(3.3-17.5)$ & \\
\hline PD-L1, cells/HPF & & & & 0.814 & & & 0.895 \\
\hline$<100$ & 66 & 14 & Not reached & & 34 & $17.9(6.9-28.8)$ & \\
\hline$\geq 100$ & 10 & 2 & Not reached & & 6 & $23.4(13.1-33.8)$ & \\
\hline PD-L2, cells/HPF & & & & 0.310 & & & 0.953 \\
\hline$<100$ & 44 & 8 & Not reached & & 24 & $23.4(12.8-33.9)$ & \\
\hline$\geq 100$ & 32 & 8 & Not reached & & 16 & $17.1(13.9-20.3)$ & \\
\hline
\end{tabular}

OS, overall survival; PFS, progression-free survival; HR, hazard ratio; CI, confidence interval; HPF, high power field.

glioblastoma, which is a completely different disease but also the malignant case in the CNS [19].

PD-L1 expression might have been induced by genetic aberrations within tumor cells such as Hodgkin lymphoma and primary mediastinal large B-cell lymphoma, which harbor amplification of 9p24.1, a genomic region that encodes PD-L1 and PD-L2. Interestingly, gain of 9p24.1 was also observed in PCNSL [7]. Indeed, PCNSL is associated with immunodeficiency [1], and PCNSL might evade the immune system utilizing PD-1 pathway [9], resulting in poorer outcome compared to non-CNS disease. Our observation suggests a possible link between high PD-1 expression and poor survival, which could explain the more aggressive behavior associated with high PD-1 expression. Furthermore, we demonstrated significant correlation of PD-1 expression with PD-L1 and PD-L2, which is in line with previous studies $[17,20]$. Therefore, our study might be used as a basis for future clinical trial targeting PD-1 pathway in PCNSL.

We acknowledge our limitation as the expression of PD-1 and PD-L1/PD-L2 have not been defined in specific cell type due to the lack of double staining with CD20, CD3 or other markers for macrophages. However, expression of PD-1 in tumor infiltrating lymphocytes and PD-L1 in tumor cells were distinguished with microscopic visualization by two independent experienced hematopathologists. Therefore, our claim that high PD-1 expression in tumor microenvironment of PCNSL is associated with poor survival is assuming that high PD-1 expression on tumor infiltrating lymphocytes in PCNSL tumor microenvironment is associated with poor survival of patients with PCNSL at diagnosis.

We also estimated PD-1, PD-L1, and PD-L2 expression visually followed by calculation of the mean
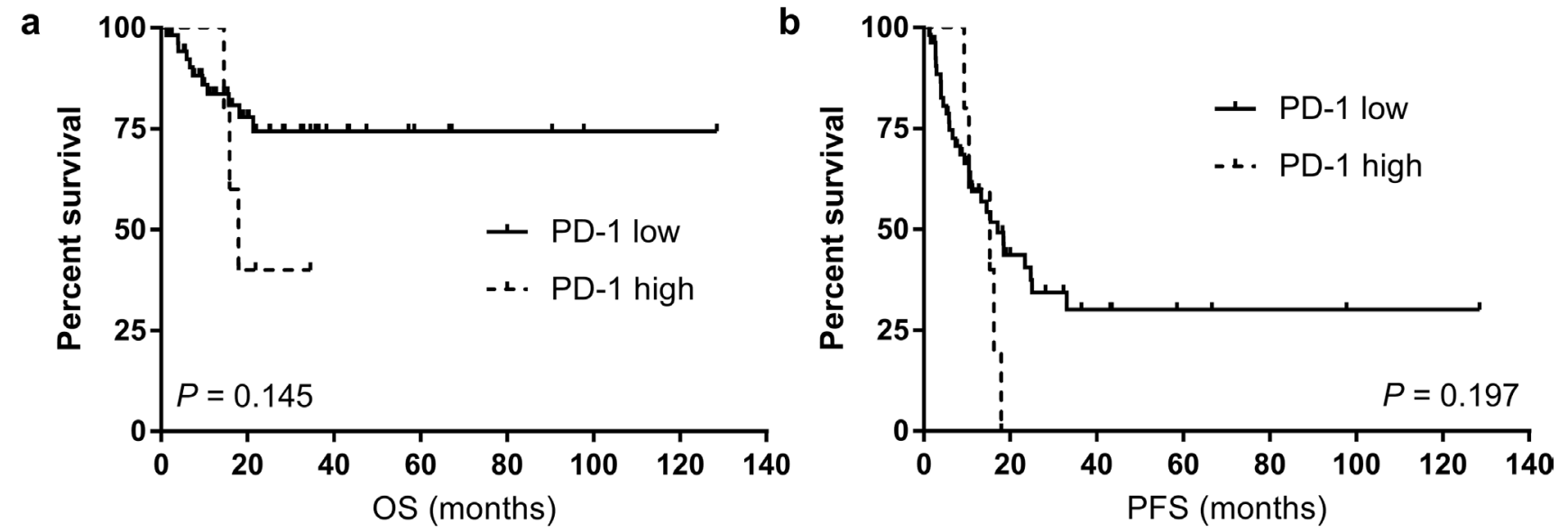

Figure 3: The overall survival (OS, a) and progression-free survival (PFS, b) according to the level of PD-1 expression among subgroup of patients who did not receive upfront autologous stem-cell transplantation (ASCT). 
Table 5: Clinical characteristics of six patients with high PD-1 expression

\begin{tabular}{|c|c|c|c|c|c|c|c|c|c|c|c|c|c|c|c|c|c|c|}
\hline & Age & Gender & $\begin{array}{l}\text { ECOG } \\
\text { PS }\end{array}$ & LDH & Lesion & $\begin{array}{c}\text { CSF } \\
\text { protein }\end{array}$ & $\begin{array}{l}\text { IELSG } \\
\text { scoring }\end{array}$ & $\begin{array}{c}\text { MSKCC } \\
\text { scoring }\end{array}$ & $\begin{array}{l}\text { PD-1, } \\
\text { cells/ } \\
\text { HPF }\end{array}$ & $\begin{array}{l}\text { PD- } \\
\text { L1, } \\
\text { cells/ } \\
\text { HPF }\end{array}$ & $\begin{array}{l}\text { PD- } \\
\text { L2, } \\
\text { cells/ } \\
\text { HPF }\end{array}$ & $\begin{array}{c}\text { Initial } \\
\text { treatment }\end{array}$ & $\begin{array}{l}\text { Interim } \\
\text { response }\end{array}$ & $\begin{array}{l}\text { Final response } \\
\text { after primary } \\
\text { chemotherapy }\end{array}$ & $\begin{array}{c}\text { Treatment } \\
\text { after primary } \\
\text { chemotherapy }\end{array}$ & $\underset{(\mathrm{mon})}{\mathrm{OS}}$ & $\begin{array}{c}\text { PFS } \\
\text { (mon) }\end{array}$ & COD \\
\hline 1 & 68 & F & 2 & Elevated & Non-deep & Normal & 3 & 2 & 92 & 10 & 0 & MVP & CR1 & $\mathrm{CR}$ & $\begin{array}{c}\text { Consolidation } \\
\text { WBRT }\end{array}$ & 15.8 & 10.4 & $\mathrm{PD}$ \\
\hline 2 & 34 & F & 1 & Normal & Deep & Elevated & 2 & 0 & 132 & 10 & 110 & MVP & CR1 & $\mathrm{CR}$ & Upfront ASCT & 8.1 & 8.1 & $\mathrm{PD}$ \\
\hline 3 & 59 & F & 1 & Normal & Non-deep & Normal & 0 & 1 & 230 & 7 & 5 & MVD & $\begin{array}{l}\text { Non- } \\
\text { CR1 }\end{array}$ & $\mathrm{CR}$ & $\begin{array}{c}\text { Consolidation } \\
\text { WBRT }\end{array}$ & 34.5 & 15.2 & Censored \\
\hline 4 & 64 & M & 1 & Normal & Deep & $\mathrm{N} / \mathrm{A}$ & N/A & 1 & 150 & 18 & 200 & MVD & $\begin{array}{l}\text { Non- } \\
\text { CR1 }\end{array}$ & $\mathrm{CR}$ & $\begin{array}{c}\text { Consolidation } \\
\text { WBRT }\end{array}$ & 17.9 & 17.9 & PD \\
\hline 5 & 39 & M & 1 & Normal & Deep & Normal & 1 & 0 & 240 & 78 & 200 & MVD & $\begin{array}{l}\text { Non- } \\
\text { CR1 }\end{array}$ & PR & $\begin{array}{c}\text { Salvage } \\
\text { Chemotherapy }\end{array}$ & 14.5 & 9.3 & PD \\
\hline 6 & 60 & $\mathrm{M}$ & 1 & Normal & Deep & Elevated & 2 & 1 & 157 & 5 & 70 & MVD & CR1 & $\mathrm{CR}$ & $\begin{array}{c}\text { Consolidation } \\
\text { WBRT }\end{array}$ & 21.8 & 16.2 & Censored \\
\hline
\end{tabular}

Abbreviations: ECOG, Eastern Cooperative Oncology Group; PS, performance status; LDH, lactate dehydrogenase; CSF, cerebrospinal fluid; EBV, Epstein-Barr virus; IELSG, International Extranodal Lymphoma Study Group; MSKCC, Memorial Sloan Kettering Cancer Center; HPF, high power field; OS, overall survival; PFS, progression-free survival; COD, cause of death; F, Female; M, Male; DLBCL, diffuse large B-cell lymphoma; N/A, not available; MVP, methotrexate, vincristine, procarbazine and dexamethasone; MVD, methotrexate, vincristine and dexamethasone; CR1, complete response after initial two cycles of chemotherapy; CR, complete response; PR, partial response; ASCT, autologous stem-cell transplantation; WBRT, whole-brain radiotherapy; PD, progressive disease.

Table 6: Correlation of PD-1 expression with PD-L1 and PD-L2

\begin{tabular}{lcc}
\hline Parameters & Correlation coefficient & $\boldsymbol{P}$ \\
\hline PD-L1 & 0.267 & 0.020 \\
PD-L2 & 0.397 & $<0.001$ \\
\hline
\end{tabular}

number of positive cells/HPF and cut-off values have been chosen by AUC of receiver operating characteristic (ROC) curve for analytic purposes. However, we acknowledge the fundamental limitation to standardize the way of determining the optimal cut-off values for PD-1 and its ligands expression from tumor biopsy samples is difficult. Therefore, the predictive relevance of our method and determining optimal cut-off values remain to be validated in future studies.

In conclusion, we showed that high PD-1 expression in PCNSL tumor microenvironment is significantly associated with inferior survival. Better knowledge of the PD-1 pathway in PCNSL, along with future trials that include PD-1 based biological risk-stratification and therapeutic targeting, are necessary for this challenging disease.

\section{MATERIALS AND METHODS}

\section{Patients}

Seventy-six biopsy-proven PCNSL patients between December 2004 and March 2016 at Severance Hospital, South Korea for whom archived formalin-fixed and paraffin-embedded (FFPE) tissue blocks at initial diagnosis were available were retrospectively analyzed. The patient cohort was previously described [6]. All the patients included in this study were immunocompetent and did not have a history of immunosuppressive drug use nor malignancies other than PCNSL. We excluded human immunodeficiency virus-related PCNSL. The diagnosis was made histologically by surgical resection or stereotactic brain biopsy. All the patients received HD-MTX-based chemotherapy as an initial treatment homogenously without receiving steroid treatment before pathologic confirm.

PCNSL was stipulated as histologically confirmed NHL confined to the CNS [1]. Baseline clinical data were retrospectively collected including age, gender, IELSG prognostic score, MSKCC prognostic score, serum EBV positivity by quantitative polymerase chain reaction, serum human immunodeficiency virus positivity by enzyme immunoassay, type of treatment including consolidative upfront ASCT and WBRT, initial response to treatment, and survival.

The ECOG performance status was determined at the time of diagnosis. Pre-treatment evaluation included contrast enhanced magnetic resonance imaging of the brain, positron emission tomography-computed tomography to exclude systemic NHL, bone marrow aspiration and biopsy with histologic, cytologic, and immunocytologic examination, ocular examination including a slit lamp examination to distinguish ocular involvement, and lumbar puncture for cerebrospinal fluid (CSF) analysis, unless contraindicated. Elevated CSF protein concentration was defined as a level more than $45 \mathrm{mg} / \mathrm{dL}$ in patients younger than 60 years of age, and a level more than $60 \mathrm{mg} / \mathrm{dL}$ in patients older than 60 years of age [2]. Involvement of deep brain structures was defined as involvement of the 
periventricular regions, basal ganglia, brainstem, and cerebellum.

The IELSG scoring system [2], which consists of five prognostic factors that are associated with poor survival in PCNSL including age $>60$ years, ECOG performance status $>1$, elevated serum lactate dehydrogenase level, elevated CSF protein concentration, and involvement of deep brain structures, was assessed in $66(86.8 \%)$ patients, as data regarding the CSF protein level were not available in $10(13.2 \%)$ patients. The MSKCC scoring system [3], consisting of age $\geq 50$ and Karnofsky performance score (KPS) $\geq 70$, which are predictors of poor outcome in PCNSL, was evaluated in all patients.

All patients included in this study had received HDMTX-based chemotherapy as an initial treatment, such as HD-MTX, vincristine, and dexamethasone (MVD, $n=$ 41) or HD-MTX, procarbazine, and dexamethasone (MVP, $n=35$ ). The MVD regimen consisted of HD-MTX 3.5 $\mathrm{g} / \mathrm{m}^{2}$ D1 delivered intravenously (i.v.) with leucovorin rescue ( $15 \mathrm{mg}$ every 6 hours, minimum 12 doses), started 24 hours after the MTX infusion, vincristine $1.4 \mathrm{mg} /$ $\mathrm{m}^{2}$ (maximum $2 \mathrm{mg}$ i.v.) D1, and dexamethasone 20 mg i.v. D1-5. The MVP regimen consisted of HD-MTX $3.0 \mathrm{~g} / \mathrm{m}^{2}$ D1 i.v. with leucovorin rescue $(15 \mathrm{mg}$ every 6 hours, minimum 12 doses), started 24 hours after the MTX infusion, vincristine $1.4 \mathrm{mg} / \mathrm{m}^{2}$ (maximum $2 \mathrm{mg}$ i.v.) D1, procarbazine $100 \mathrm{mg} / \mathrm{m}^{2} \mathrm{D} 1-14$ given orally, and dexamethasone $20 \mathrm{mg}$ i.v. D1-14. Tumor response was assessed according to the criteria of the International PCNSL Collaborative Group [21]. Treatment response was recorded as complete response1 (CR1), if the patients achieved a CR (disappearance of all enhancing abnormalities on brain magnetic resonance imaging) after initial induction HD-MTX-based chemotherapy. The institutional review board of Severance Hospital approved this study.

\section{Immunohistochemistry of PD-1, PD-L1, and PD-L2}

Immunohistochemical staining for PD-1 (clone NAT105; dilution 1:100; Abcam, Cambridge, UK), PD-L1 (clone ab58810; dilution 1:100; Abcam, Cambridge, UK), and PD-L2 (clone MIH18; dilution 1:50; Sigma-Aldrich, St. Louis, MO, USA) using archived FFPE tissue slides were performed according to the protocols for automated immunohistochemistry using the Ventana Discovery XT automatic platform (Ventana Automated Systems, Tucson, AZ, USA).

\section{Quantitative assessment of PD-1, PD-L1, and PD-L2}

The stained sections were screened at $40 \times$ magnification via Olympus BX51 microscope (Olympus,
Deutschland GmbH, Hamburg, Germany; 40× objective, $10 \times$ ocular, and $0.55 \mathrm{~mm}$ ocular diameter) to identify the areas with the most abundant positive cells within the tumor area. Six representative fields with the highest staining tumor cells were selected at a magnification of $400 \times \mathrm{HPF}$. We analyzed $1.44 \mathrm{~mm}^{2}$ per case because the area of a single image was $0.24 \mathrm{~mm}^{2}$. PD-1 positive tumor-infiltrating mononuclear cells (i.e. lymphocytes and macrophages) and PD-L1/PD-L2 positive tumor cells were counted manually, and the mean numbers of positive cells/HPF were calculated. Blinded quantitative assessments were performed by two independent pathologists (SHK and W-IY). The cut-off values for high PD-1, PD-L1, and PD-L2 were evaluated by the AUC of the ROC curve, and we established cut-offs of 70 cells/HPF for PD- 1 and 100 cells/HPF for PD-L1/PDL2. Therefore, high PD-1 expression was defined as $\geq 70$ cells/HPF, and high PD-L1/PD-L2 were defined as $\geq 100$ cells/HPF.

\section{Statistical analysis}

OS was defined as the time from initial diagnosis to death or last follow-up; PFS was defined as the time from initial diagnosis to relapse, disease progression, or death. Patient data were collected retrospectively until March 2016, and patients who were alive at the last follow-up contact were censored. OS and PFS were plotted using the Kaplan-Meier method and compared using the log-rank test. Analysis of categorical variables was performed using the chi-squared test or Fisher's exact test. The Wilcoxon rank-sum test was used for continuous variables. The Cox regression model was used in both univariate and multivariate analysis. All $P$ values were two-sided, and $P$ $<0.05$ was considered statistically significant. Statistical analyses were performed using SPSS for Windows (Version 20.0, IBM Corp., Chicago, IL, USA).

\section{Author contributions}

Hyunsoo Cho and Se Hoon Kim acquired, analyzed the data and wrote the manuscript. Jin Seok Kim conceived the study, interpreted the data, and critically revised the manuscript. Se Hoon Kim and Woo-Ick Yang interpreted pathological and immunohistochemical findings. SooJeong Kim, Jong Hee Chang, Chang-Ok Suh, Yu Ri Kim, Ji Eun Jang, June-Won Cheong and Yoo Hong Min participated in data acquisition and comprehensive discussion.

\section{ACKNOWLEDGMENTS}

This work was presented in the form of e-poster presentation at the 21th Congress of the European Hematology Association, Copenhagen, Denmark, June 9-12, 2016. 


\section{CONFLICTS OF INTEREST}

The authors have no conflicts of interest.

\section{GRANT SUPPORT}

This work was supported by a faculty research grant, Yonsei University College of Medicine, 2015 (62015-0029).

\section{REFERENCES}

1. Deckert M, Engert A, Bruck W, Ferreri AJ, Finke J, Illerhaus G, Klapper W, Korfel A, Kuppers R, Maarouf M, Montesinos-Rongen M, Paulus W, Schlegel U, et al. Modern concepts in the biology, diagnosis, differential diagnosis and treatment of primary central nervous system lymphoma. Leukemia. 2011; 25:1797-1807.

2. Ferreri AJ, Blay JY, Reni M, Pasini F, Spina M, Ambrosetti A, Calderoni A, Rossi A, Vavassori V, Conconi A, Devizzi L, Berger F, Ponzoni M, et al. Prognostic scoring system for primary CNS lymphomas: the International Extranodal Lymphoma Study Group experience. J Clin Oncol. 2003; 21:266-272.

3. Abrey LE, Ben-Porat L, Panageas KS, Yahalom J, Berkey B, Curran W, Schultz C, Leibel S, Nelson D, Mehta M, DeAngelis LM. Primary central nervous system lymphoma: the Memorial Sloan-Kettering Cancer Center prognostic model. J Clin Oncol. 2006; 24:5711-5715.

4. Lopez-Girona A, Heintel D, Zhang LH, Mendy D, Gaidarova S, Brady H, Bartlett JB, Schafer PH, Schreder M, Bolomsky A, Hilgarth B, Zojer N, Gisslinger H, et al. Lenalidomide downregulates the cell survival factor, interferon regulatory factor-4, providing a potential mechanistic link for predicting response. Br J Haematol. 2011; 154:325-336.

5. Braaten KM, Betensky RA, de Leval L, Okada Y, Hochberg FH, Louis DN, Harris NL, Batchelor TT. BCL-6 expression predicts improved survival in patients with primary central nervous system lymphoma. Clin Cancer Res. 2003; 9:1063-1069.

6. Cho H, Kim SH, Kim SJ, Chang JH, Yang WI, Suh CO, Cheong JW, Kim YR, Lee JY, Jang JE, Kim Y, Min YH, Kim JS. The prognostic role of CD68 and FoxP3 expression in patients with primary central nervous system lymphoma. Ann Hematol. 2017; 96:1163-1173.

7. Tun HW, Personett D, Baskerville KA, Menke DM, Jaeckle KA, Kreinest P, Edenfield B, Zubair AC, O'Neill BP, Lai WR, Park PJ, McKinney M. Pathway analysis of primary central nervous system lymphoma. Blood. 2008; 111:3200-3210

8. Ponzoni M, Berger F, Chassagne-Clement C, Tinguely M, Jouvet A, Ferreri AJ, Dell'Oro S, Terreni MR, Doglioni C, Weis J, Cerati M, Milani M, Iuzzolino P, et al. Reactive perivascular T-cell infiltrate predicts survival in primary central nervous system B-cell lymphomas. Br J Haematol. 2007; 138:316-323.

9. Chapuy B, Roemer MG, Stewart C, Tan Y, Abo RP, Zhang L, Dunford AJ, Meredith DM, Thorner AR, Jordanova ES, Liu G, Feuerhake F, Ducar MD, et al. Targetable genetic features of primary testicular and primary central nervous system lymphomas. Blood. 2016; 127:869-881.

10. Topalian SL, Taube JM, Anders RA, Pardoll DM. Mechanism-driven biomarkers to guide immune checkpoint blockade in cancer therapy. Nat Rev Cancer. 2016; 16:275-287.

11. Ansell SM, Lesokhin AM, Borrello I, Halwani A, Scott EC, Gutierrez M, Schuster SJ, Millenson MM, Cattry D, Freeman GJ, Rodig SJ, Chapuy B, Ligon AH, et al. PD-1 blockade with nivolumab in relapsed or refractory Hodgkin's lymphoma. N Engl J Med. 2015; 372:311-319.

12. Nayak L, Iwamoto FM, LaCasce A, Mukundan S, Roemer MG, Chapuy B, Armand P, Rodig SJ, Shipp MA. PD-1 blockade with nivolumab in relapsed/refractory primary central nervous system and testicular lymphoma. Blood. 2017; 129:3071-3073. https://doi.org/10.1182/ blood-2017-01-764209.

13. Wang CC, Carnevale J, Rubenstein JL. Progress in central nervous system lymphomas. Br J Haematol. 2014; 166:311-325.

14. Paydas S, Bagir E, Seydaoglu G, Ercolak V, Ergin M. Programmed death-1 (PD-1), programmed death-ligand 1 (PD-L1), and EBV-encoded RNA (EBER) expression in Hodgkin lymphoma. Ann Hematol. 2015; 94:1545-1552.

15. Myklebust JH, Irish JM, Brody J, Czerwinski DK, Houot R, Kohrt HE, Timmerman J, Said J, Green MR, Delabie J, Kolstad A, Alizadeh AA, Levy R. High PD-1 expression and suppressed cytokine signaling distinguish $\mathrm{T}$ cells infiltrating follicular lymphoma tumors from peripheral $\mathrm{T}$ cells. Blood. 2013; 121:1367-1376.

16. Berghoff AS, Ricken G, Widhalm G, Rajky O, Hainfellner JA, Birner P, Raderer M, Preusser M. PD1 (CD279) and PD-L1 (CD274, B7H1) expression in primary central nervous system lymphomas (PCNSL). Clin Neuropathol. 2014; 33:42-49.

17. Four M, Cacheux V, Tempier A, Platero D, Fabbro M, Marin G, Leventoux N, Rigau V, Costes-Martineau V, Szablewski V. PD1 and PDL1 expression in primary central nervous system diffuse large B-cell lymphoma are frequent and expression of PD1 predicts poor survival. Hematol Oncol. 2016. https://doi.org/10.1002/hon.2375.

18. Cho H, Chang JH, Kim YR, Kim SJ, Chung H, Park H, Lee JY, Jang JE, Kim Y, Kim SH, Yang WI, Suh CO, Cheong JW, et al. The role of upfront autologous stem cell transplantation in high-risk younger patients with primary central nervous system lymphoma. Br J Haematol. 2016; 174:444-453.

19. Berghoff AS, Kiesel B, Widhalm G, Rajky O, Ricken G, Wohrer A, Dieckmann K, Filipits M, Brandstetter A, Weller M, Kurscheid S, Hegi ME, Zielinski CC, et 
al. Programmed death ligand 1 expression and tumorinfiltrating lymphocytes in glioblastoma. Neuro Oncol. 2015; 17:1064-1075.

20. Taube JM, Klein A, Brahmer JR, Xu H, Pan X, Kim JH, Chen L, Pardoll DM, Topalian SL, Anders RA. Association of PD-1, PD-1 ligands, and other features of the tumor immune microenvironment with response to anti-PD-1 therapy. Clin Cancer Res. 2014; 20:5064-5074.
21. Abrey LE, Batchelor TT, Ferreri AJ, Gospodarowicz M, Pulczynski EJ, Zucca E, Smith JR, Korfel A, Soussain C, DeAngelis LM, Neuwelt EA, O'Neill BP, Thiel E, et al. Report of an international workshop to standardize baseline evaluation and response criteria for primary CNS lymphoma. J Clin Oncol. 2005; 23:5034-5043. 